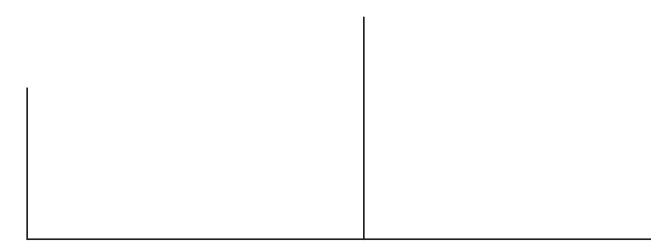

Rev. Latinoam. Psicopat. Fund., III, 4, 112-121

\title{
Psicopatologia Fundamental: de una cierta transmision
}

\author{
Flora Singer
}

\begin{abstract}
La psicopatología fundamental, conformada a partir del trabajo universitario y teórico de Pierre Fédida, se constituye como punto de encuentro de diferentes disciplinas en relación al sufrimiento humano, y plantea interrogantes metodológicas, entre otras, cómo establecer un diálogo que articule distintos recortes teóricos, y de qué manera tratar las diferencias relativas. El tema de las diferencias está presente tanto por el lado de los métodos de investigación y de intercambio, como por el de la concepción de una estructura institucional que acoja esas diferencias.

El psicoanálisis puede hacer contribuciones a estos temas, en la medida en que se ve confrontado a una doble alteridad: tópica la relación conciencia/inconsciente - e intersubjetiva, ya que es condición del dispositivo analítico poder acoger la subjetividad del otro en tanto radicalmente otro.

Hay una homología estructural entre los niveles intrapsíquico, intersubjetivo, interteórico e interdisciplinario en lo que hace a una epistemología de la alteridad.
\end{abstract}

* Trabajo presentado en el V Congresso Brasileiro de Psicopatologia Fundamental, de 15 a 17 de setiembre de 2000, en Campinas, SP. 


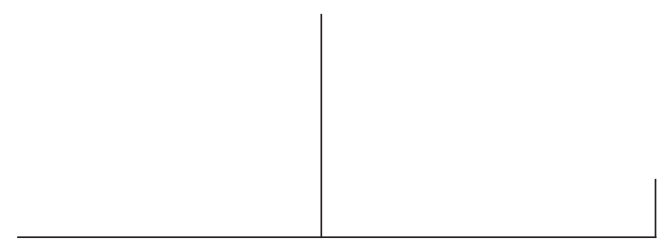

El saber en psicoanálisis escapa a toda tentativa de institucionalización pues es propio a la alteridad de su objeto resistir y dejar siempre un resto. De manera similar, la psicopatología pundamental tampoco se adecua a una institucionalización del saber. Es un espacio vacío - khôra - que acoge movimientos metafóricos inherentes a la producción de saber, y al no-saber que en forma complementaria lo acompaña.

El "nombre del padre" opera así, no como fidelidad a una letra, sino como habilitación a la creación y soporte de lo figurable a venir, a partir del acto fundacional de khôra.

Palabras clave: Psicopatología fundamental, pathos, alteridad, epistemología

Dar un nombre, ¿es aún dar? ¿es dar algo? Y siempre otra cosa que un nombre propio (...) el don del nombre da lo que no tiene, lo cual es quizás, sobre todo, la esencia, o sea, más allá del ser, la inesencia del don.

Jacques Derrida, Salvo el nombre

¿Qué se me pide exactamente? ¿Si pienso antes de clasificar? ¿Si clasifico antes de pensar? ¿Cómo clasifico lo que pienso? ¿Cómo pienso cuando quiero clasificar?(...) Es tan tentador querer distribuir el mundo entero de acuerdo a un código único; una ley universal regiría el conjunto de los fenómenos: dos hemisferios, cinco continentes, masculino y femenino, animal y vegetal, singular plural, derecha izquierda, cuatro estaciones, cinco sentidos, seis vocales, siete días, doce meses, veintiséis letras. Lamentablemente no funciona, jamás ha comenzado ni siquiera a funcionar, no funcionará jamás. Ello no impide que no obstante se continuará aún por largo tiempo a categorizar tal o cual animal según posea un número impar de dedos o cuernos huecos.

Georges Perec, Pensar/Clasificar

Las nuevas fronteras y desafíos de la psicopatología, tienen que ver con cambios de paradigmas que alteran los juegos de fuerzas intercientíficos. Nuevos avances en la biología, las neurociencias, la farmacología, inducen un reacomodamiento en otras disciplinas. Desde el psicoanálisis surge la reflexión acerca de su lugar en este reordenamiento, 
y por otra parte, la necesidad de un diálogo interdisciplinario con los otros campos y la clarificación de las fronteras y metodologías específicas.

Es en este contexto que se ubica la creación, por parte del prof. Pierre Fédida hace ya algunos años, del Laboratorio de Psicopatología Fundamental y Psicoanálisis de la Universidad Paris 7.

La psicopatología fundamental surge a partir del trabajo universitario y teórico de P. Fédida. Esta propuesta, promueve el aggiornamiento del psicoanálisis con nuevos esquemas nosográficos, avances científicos, y cambios sociales. A pesar de que la psicopatología fundamental deriva del campo psicoanalítico, no se confunde con él, pues de lo que se trata es, además de un trabajo interno al campo psicoanalítico, de la puesta en relación del psicoanálisis con lo otro fuera de su campo: otras disciplinas, metodologías, nuevas coyunturas culturales y sociales.

Hay en el paradigma de saber de nuestra época, y no sólo en relación a la psicopatología, un doble movimiento, de globalización de los campos de saber por un lado, pero tambien de afinamiento de las singularidades en juego. Ello lleva a la necesidad de definir los campos específicos, pero también a admitir en el análisis las mezclas, y el estudio de las zonas de pasaje e intersección entre distintos campos. Se crea así la necesidad de una epistemología que tenga en cuenta la puesta en relación con lo otro.

La problemática de la alteridad es central en esta nueva distribución del saber sobre el pathos, conforma una episteme específica al psicoanálisis, y puede constituir su aporte en este contexto.

El psicoanálisis se ve confrontado a una doble alteridad: tópica, en la relación conciencia/inconsciente donde el inconsciente conserva su irreductibilidad, e intersubjetiva, ya que el dispositivo psicoanalítico considera la subjetividad del sujeto como siendo radicalmente otro. En ese sentido el psicoanálisis es paradigmático de una posición epistémica que jerarquiza el mantenimiento de las diferencias, a diferencia de una posición epistémica tradicional que busca elementos universalizables y leyes generales.

La búsqueda de identidades y leyes universales ha sido y sigue siendo, una aspiración científica de corte positivista. La insuficiencia de este abordaje se pone de manifiesto cuando se aplica a las llamadas ciencias humanas, ciencias de lo singular, lo heteróclito e indeterminado. La preeminencia histórica de criterios positivistas ha saldado la cuestión negando el estatuto de cientificidad a las paradojalmente llamadas ciencias humanas, sin por ello resolver el estatuto de las realidades complejas con las que éstas están comprometidas, como en el caso de la psicopatología.

Un aspecto del debate actual en psicopatología reedita estos viejos debates y recoge estas dificultades. Un trabajo reciente del prof. Mário Eduardo Costa Pereira 


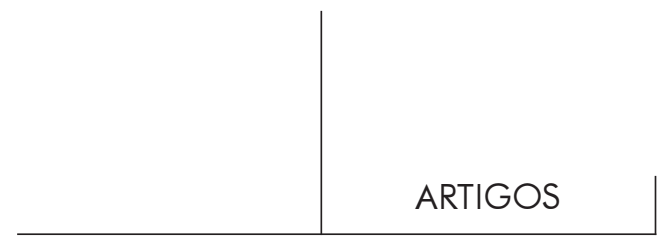

sobre el DSM lo pone de manifiesto. Los DSM son sistemas de clasificación de los trastornos mentales que apuntan a una operatividad y un pragmatismo que pueden ser efectivos en un cierto corte metodológico, a condición de renunciar a una posición hegemónica en el abordaje del fenómeno psicopatológico, y reconocer lo que excede a dicho sistema. Lo que excede no es poca cosa:

- La dimensión subjetiva del individuo no coincide con lo objetivamente constatable.

- La singularidad del pathos, y lo indecidible de realidades complejas, no pueden ser recogidos en leyes generales.

- Esas realidades complejas son incompatibles con lenguajes definidos o formalizados, y con convencionalismos nosográficos basados en un ideal de uniformización del lenguaje.

El pathos habla de lo subjetivo, lo singular, lo complejo, lo indeterminado, y también de la dificultad del lenguaje para abordarlo. Esto hace necesaria otro tipo de episteme. En lo intersticial de una episteme de lo positivo, el psicoanálisis tiene algo para decir acerca de esto que escapa. El mismo movimiento que deja afuera al psicoanálisis de un cierto abordaje psicopatológico, circunscribe también su especificidad y su contribución a otro tipo de abordaje.

El psicoanálisis habla de lo otro intrapsíquico e intersubjetivo, y esas alteridades presentan una homología estructural con el otro interteórico e interdisciplinario que aborda la psicopatología fundamental.

Esta línea filiatoria que une a la psicopatología fundamental con el psicoanálisis coloca a este último en una posición paradigmática, pero no en el sentido de un modelo hegemónico, sino en el sentido de un proveedor de una episteme de la alteridad que hace posible la reunión de diferentes teorías en torno al pathos, al mismo tiempo que la conservación de sus alteridades relativas. El psicoanálisis, además de ser una disciplina más, provee el espacio que habilita a ese intercambio.

Es condición del objeto del psicoanálisis no reducirse a su teorización, en la medida en que siempre un plus enigmático resiste e insiste. Las dificultades que tienen las propias teorías psicoanalíticas de ponerse en relación, hablan de ello. También la historia del movimiento psicoanalítico muestra la inadecuación de instituciones estáticas, con aspiraciones a transmisiones claras. Las escisiones muestran desde lo real, la imposibilidad de decirlo todo acerca del inconsciente. Pero entonces, ¿desde qué lugar da su contribución el psicoanálisis?

Más que las instituciones o las distintas teorías, es el lugar vacío del dispositivo de la cura el que provee el espacio para que los saberes advengan, se transformen y circulen en relación a una alteridad. Pierre Fédida denomina khôra a ese espacio donde algo del orden de un sentido nuevo se genera, tomando el término de El Timeo de Platon, y de la utilización que de él hace Jacques Derrida. 


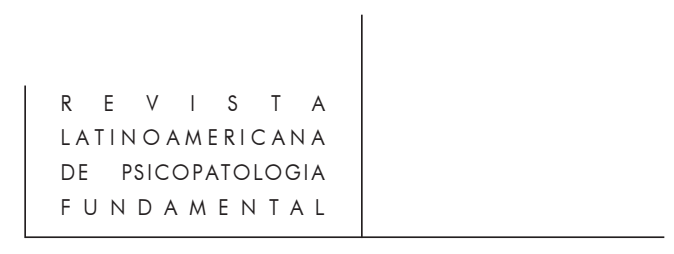

El dispositivo de la Psicopatología Fundamental es también un lugar vacío, como khôra, donde una pluralidad de puntos de vista en relación al pathos advienen y se transforman por obra de su propia puesta en relación y movimiento.

Que en ese lugar vacío algo del orden del saber adviene, significa que se rescata en el orden del saber: 1) el momento de producción y de transformación del saber, y no sólo el saber sabido; 2) el reconocimiento de lo intersticial, de lo nuevo, de lo otro, y la cuota de incerteza que ello conlleva; 3) la aceptación de las contradicciones dinámicas que el lenguaje tiende a presentar como falsas oposiciones.

El orden filiatorio de la psicopatología fundamental tiene que ver con esta dialéctica entre positividad y negatividad, entre un lugar de saber y un espacio vacío donde nuevos saberes pueden advenir.

J. Derrida distingue entre el nombre común y el nombre propio, y dice que el nombre propio no pertenece propiamente a la lengua, y sólo puede inscribirse en ella dejándose traducir, o sea interpretar en su equivalente semántico. Pero a partir de ese momento no puede ser más considerado nombre propio.

La psicopatología fundamental como nombre común es contenido semántico, cuyo análisis el prof. Manoel Berlinck ha abordado en sus importantes contribuciones que restituyen la dimensión esencialmente subjetiva del pathos, su condición antropológica, y la compleja dialéctica entre lo normal y lo patológico.

Pero la psicopatología fundamental es también un nombre propio, el "nombre del padre", en donde PF de Psicopatología Fundamental es también PF de Pierre Fédida. En ese caso no connota un saber, porque el nombre propio no connota saberes, sólo recoge un orden filiatorio sin deudas, sin testamentos o sentidos predeterminados. Sólo "crea un lugar en el lenguaje" (Pierre Fédida). Un lugar que, si el padre cumple su función, debe quedar vacío para que sus hijos, por la misma ley de filiación, puedan crear otros saberes. Es menos transmisión de saber y fidelidad a una letra, que don de un lugar y habilitación a la creación. Se establece así el corte y la diferencia con el padre por la cual algo nuevo puede siempre advenir.

¿Cuál es la ley de regulación de ese lugar vacío donde los distintos saberes en torno al pathos se producen, circulan, se ponen en relación? Esta regulación presenta aspectos metodológicos y de validación en relación a los cuales se imponen algunas reflexiones.

A nivel metodológico se impone la pregunta de cómo establecer un diálogo que articule distintos recortes teóricos, y de qué manera tratar las diferencias relativas.

Si lo otro, lo otro de la otra teoría, la otra disciplina, se respeta como alteridad, es esta alteridad la que debemos aprender a interrogar, sin reducciones ni clasificaciones, en una puesta en relación de semejanzas y diferencias.

Hay disciplinas que no se mueven dentro de lenguajes bien definidos, sino por aproximaciones, metáforas, que no se adecúan totalmente a sus objetos o sufren 


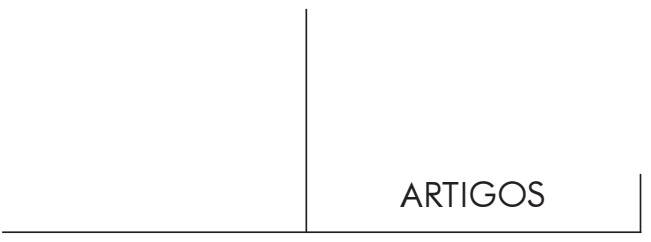

transformaciones diacrónicas, como en psicoanálisis. Esos lenguajes también generan un plus, que hace que la zona de intersección entre disciplinas sea más una zona de diseminación del propio lenguaje, que un espacio bien definido.

Qué quiere decir sexualidad, placer, histeria, sueño, dentro de campos teóricos distintos o aún en la diacronía de una teoría, sólo puede ser abordado desde una lógica dinámica y de lo intersticial. Se trata de entes en negativo, en el sentido de que no tienen realidad empírica, y en esa medida toda teoría no sólo transporta saber, sino que precipita, configura al propio objeto en el plano del lenguaje, y se constituye como soporte figurativo del mismo.

Tanto por el tipo de objetos, como por el trabajo del lenguaje, todas las zonas de pasaje involucradas dejan restos: los pasajes entre los modelos utilizados y la cosa; entre las definiciones relativas de los términos, entre las fronteras de cada disciplina, son zonas de intercambios no del todo determinados, donde algo escapa, del lado del objeto $y$ del lado del lenguaje. Los puntos ciegos son activos: pueden generar saber y también contaminación, y en todo caso constituyen un núcleo a interrogar. El vacío no es ausencia, es presencia activa.

Del lado del objeto, la sumatoria de los campos en psicopatología, no construye el objeto. Este sigue siendo un cuasi-objeto, como lo denomina Francisco Varela en un trabajo reciente.

Del lado del lenguaje, cuando se habla no se emplea el mismo lenguaje, y toda apropiación de saber para el propio campo implica una transformación de sentido. No hay incorporación directa de saber ni traducción directa de una lengua a otra. Cuando la histeria pasa del campo psiquiátrico al psicoanalítico, hay allí una transvaluación que la transforma en otra noción, esta vez interna al campo psicoanalítico, sólo que el pasaje entre una noción y otra es del orden de las différances: hay algo de lo mismo y algo de lo nuevo.

Cuando estos saberes parciales se ponen en relación los parámetros de lo verdadero-falso no son los que regulan estos intercambios. Isabelle Stengers considera que "ninguna proposición científica puede ser declarada verdadera si no ha interesado", y recuerda que etimológicamente interesar es situarse entre, en un sentido activo, en donde algo, dispositivo, razonamiento, "pueda concernir a otros", situarse "entre mi trabajo y el de aquel que lo propone" y eventualmente transformar así mi propio trabajo. Para Stengers una proposición que no interesa a nadie no es ni verdadera ni falsa; sólo "forma parte del ruido que acompaña las actividades científicas".

Todo relacionamiento con lo otro, aún a nivel de interrogación, a nivel, como dice Fédida, del interés que puede despertar la orilla opuesta, puede ser productiva y transformativa en el interior del propio campo, puede promover la creación de nuevas metáforas para acercarse a lo que escapa del objeto. Una actitud epistémica más sensible a las diferencias que a las grandes síntesis puede promover la disponibilidad de acoger lo intersticial del sentido aún no encontrado. 


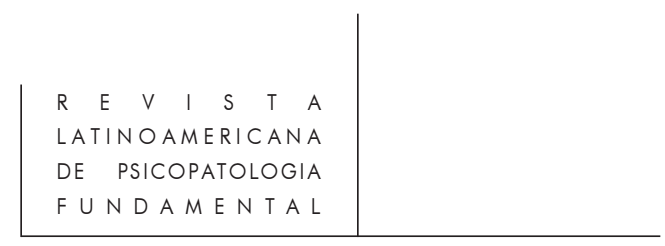

A nuestro entender uno de los numerosos desafíos de la psicopatología fundamental es metodológico: el de cómo crear un lugar epistémico adecuado a sus objetos y disciplinas, y al lado de una epistemología de tipo empírico pragmático, restituir la dimensión negativa de lo que resiste a ser dicho, y transcurre en la intersticialidad de los saberes.

Este desafío en el terreno teórico, se acompaña de otro similar a nivel clínico, nuevamente qué lugar asignarle a lo otro. Lo otro diferente a la positividad y la representación.

El psicoanálisis surge en un momento en donde un orden social estructurado provee sentidos asignados, identificaciones estables, roles definidos, en donde muchas veces la histérica sufre de ese exceso representacional, ese exceso de simbólico que comprime su cuerpo y su sexualidad.

La cultura actual presenta una fragmentación en lo simbólico, desgarramientos de valores, de creencias, de soportes identificatorios estables, que llevan a la pérdida de sentido o a cambios vertiginosos de sentido.

No es casual que se hable de pánico, desamparo, depresiones, toxicomanías, como algunos de los males de nuestra época. No es casual tampoco que las relaciones de objeto que acompañan estos sufrimientos sean difusas, y transcurran en los límites de lo representable.

Al mismo tiempo se promueven actos terapéuticos eminentemente positivos: las nosografías ya mencionadas, terapias breves, actos médicos altamente tecnificados, psicofármacos sofisticados. Paradojalmente a mayor negatividad del sufrimiento se corresponde un plus de positividad en el acto terapéutico, que muchas veces se constituye como un intento fallido de resolución, que obtura la posibilidad de recoger lo a-representacional como tal, lo que aún no puede ser dicho. Eso que transcurre en los intersticios del sentido o de la representación, tiene un lugar terapéutico, el de poder trasladarlo al plano del lenguaje sin positivizarlo, y poder darle una figurabilidad adecuada. La coyuntura teórico-clínica actual necesita de una sensibilidad apropiada de la teoría para recibirla.

Este espacio de reflexión que es la psicopatología fundamental, que induce a pensar la coyuntura actual de la psicopatología, compromete la teoría, la clínica, y también nuestro propio dispositivo de pensar. Como dice Michel Foucault se trata de "penser autrement", que admite una doble traducción: cómo pensar lo otro, y también cómo pensar de otra manera.

\section{Referências Bibliográficas}

Berlinck, M. Catástrofe e representação: notas para uma teoria geral da psicopatologia fundamental. Revista Latinoamericana de Psicopatologia Fundamental. São Paulo: Escuta. v. II, n. 1, 1999. 


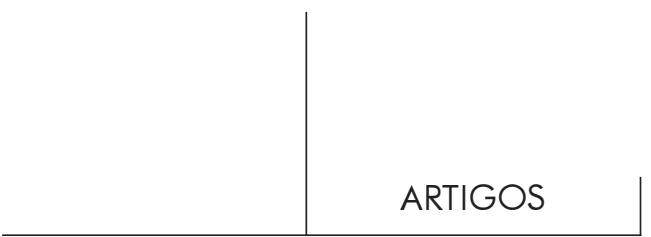

Cohen, A.; VARELA, F. Facing up to the embarrassment. The practice of subjectivity in neuroscientific and psychoanalytic experience. Trabajo presentado en Etats Généraux de la Psychanalyse, Paris, 2000.

Costa Pereira, M. E. A paixão nos tempos do DSM: sobre o recorte operacional do campo da psicopatologia. Trabajo presentado en Etats Généraux de la Psychanalyse, Paris, 2000.

Derrida, J. Des tours de Babel. In Psyché, inventions de l'autre. Galilée: Paris, 1987.

FÉDIDA, P. Discussion de l'article de D.Widlöcher Neurobiologie et psychanalyse. Les opérateurs de commutation. Revue Internationale de Psychopathologie. PUF: Paris, n. 2, 1990.

Le site de l'étranger. La situation psychanalytique. Paris: PUF, 1995. (Em português: $O$ sítio do estrangeiro. A situação psicanalítica. São Paulo: Escuta, 1996.)

, Lacoste, P. Psychopathologie/Métapsychologie. La fonction des points de vue. Revue Internationale de Psychopathologie. Paris: PUF, n. 8, 1992.

Singer, F. Modelos en psicoanálisis. Revista Latinoamericana de Psicopatologia Fundamental. São Paulo: Escuta. v. I, n. 2, 1998.

La teoría y su objeto, a ser publicado en "A Pesquisa em Psicopatología Fundamental", Ed. Escuta, Sao Paulo, 2000.

Stengers, I. Boîtes noires scientifiques, boîtes noires professionnelles. In La psychanalyse, une science? Paris: Les Belles Lettres, 1989.

\section{Resumos}

A psicopatologia fundamental, configurada a partir do trabalho universitário $e$ teórico de Pierre Fédida, constitui-se como ponto de encontro de diferentes disciplinas relacionadas com o sofrimento humano, e suscita interrogantes metodológicos, entre outros, como estabelecer um diálogo que articule distintos recortes teóricos, e de que forma tratar as diferenças relativas. $O$ tema das diferenças está presente tanto pelo lado dos métodos de investigação e de intercâmbio, como pelo da concepção de uma estrutura institucional que acolha essas diferenças.

A psicanálise pode contribuir com estes temas, à medida que se vê confrontada com uma dupla alteridade: tópica - a relação consciência/inconsciente - e intersubjetiva, já que é condição do dispositivo analítico poder acolher a subjetividade do outro enquanto radicalmente outro.

Existe uma homologia estrutural entre os níveis intrapsíquico, intersubjetivo, interteórico e interdisciplinar no que faz uma epistemologia da alteridade.

O saber em psicanálise escapa a toda tentativa de institucionalização, pois é próprio da alteridade de seu objeto resistir e deixar sempre um resto. De maneira si- 
milar, a psicopatologia fundamental tampouco se adequa a uma institucionalização do saber. É um espaço vazio - khôra - que acolhe movimentos metafóricos inerentes à produção de saber, e ao não-saber, que de forma complementar o acompanha.

$O$ "nome-do-pai" opera, assim, não como fidelidade a uma letra, mas como habilitação à criação e suporte do figurável a vir, a partir do ato fundacional de khôra.

Palavras-chave: Psicopatologia fundamental, pathos, alteridade, epistemologia

La psychopathologie fondamentale, créée à partir du travail universitaire et théorique de Pierre Fédida, se constitue comme point de rencontre de différentes disciplines autour de la souffrance humaine, et elle pose des questions méthodologiques, entre autres, comment un dialogue peut s'établir qui puise articuler des coupures théoriques différentes et de quelle facon traiter les différences relatives. Le sujet des différences est présent aussi bien du côté des méthodes de recherche et d'échange, que du côté de la conception d'une structure institutionnelle qui accueille ces différences.

La psychanalyse peut faire ses contributions à ces sujets, dans la mesure où elle se voit confrontée à une double altérité, topique - le rapport consciencelinconscient - et intersubjective, puisqu'une des conditions du dispositif psychanalytique est celle de pouvoir accueillir la subjectivité de l'autre en tant que radicalement autre.

Il y a une homologie structurelle entre les niveaux intrapsychique, intersubjectif, interthéorique et interdisciplinaire, en ce qui concerne une épistémologie de l'altérité.

Le savoir en psychanalyse échappe à toute tentative d'institutionalisation, car l'altérité de son objet résiste et laisse toujours un reste. D'une facon similaire, la Psychopathologie Fondamentale ne s'adapte pas non plus à une institutionalisation du savoir. C'est un espace vide - telle khôra - qui accueille des mouvements métaphoriques inhérents à la production de savoir, et au non-savoir qui, de facon complémentaire, l'accompagne.

Le "nom du père" opère ainsi, non pas comme fidelité à une lettre, mais comme habilitation à la création et support du figurable à venir, à partir de l'acte fondationnel de khôra.

Mots clés: Psychopathologie Fondamentale, pathos, altérité, épistémologie.

The Fundamental Psychopathology, created through the universitary and theoretic work of Pierre Fédida, is a meeting point of different disciplines engaged with human sufferance, and some methodological questions therefrom arise, among others, how to establish a dialogue between different theories and how to treat relative differences. The subject of differences concerns both, the point of view of research and exchange methods, as well as the conception of an adequate institutional structure for receiving these differences.

Psychanalysis can make some contributions to these subjects, as it deals with a double alterity: topical - the conscious/unconscious relationship - and intersubjective, 


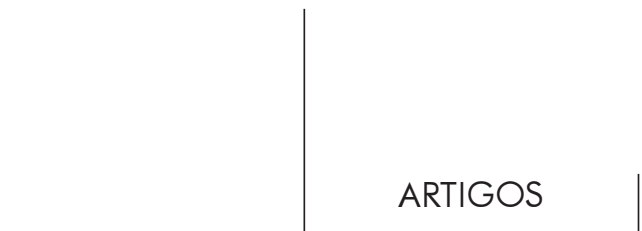

because one of the main conditions of the analytic dispositive is the capacity of receiving subjectivity in its radical otherness.

There is a structural homology between intrapsychic, intersubjectif, intertheoretic and interdisciplinary levels: they are all concerned by an otherness epistemology.

Psychoanalytic knowledge does not adapt to institutionalisation attempts. The otherness of its object offers resistances, and a remainder is always left. Similarly, Fundamental Psychopathology does not adapt to a knowledge institutionalisation. Is is an empty space - khôra - which receives metaphoric mouvements coming from knowledge production as well as un-knowledge which naturally accompanies that production.

"Father's name" does not therefore represent fidelity to a given knowledge, but habilitation to creation and support of outcoming metaphores, in khôra, which is both, empty space and foundation act.

Key words: Fundamental Psychopathology, pathos, alterity, epistemology.

Versão inicial recebida em setembro de 2000.

Versão revisada recebida em novembro de 2000. 